\title{
Multiuser Beamforming with Limited Feedback for FDD Massive MIMO Systems
}

\author{
Senyao Zheng, Hui Zhao, Long Zhao, Jie Mei, and Weimin Tang \\ Wireless Signal Processing \& Network (WSPN) Lab, BUPT, Key Laboratory of Universal Wireless Communication, \\ Ministry of Education, Beijing 100876, China
}

Correspondence should be addressed to Senyao Zheng; zhengsy12@gmail.com

Received 29 September 2015; Accepted 14 February 2016

Academic Editor: Jilei Hou

Copyright (C) 2016 Senyao Zheng et al. This is an open access article distributed under the Creative Commons Attribution License, which permits unrestricted use, distribution, and reproduction in any medium, provided the original work is properly cited.

This paper discusses the multiuser beamforming in FDD massive MIMO systems. It first introduces the feature of FDD massive MIMO systems to implement multiuser beamforming schemes. After that, considering the realistic implementation of multiuser beamforming scheme in FDD massive MIMO systems, it introduces the knowledge of channel quantization. In the main part of the paper, we introduce two traditional multiuser beamforming schemes and analyse their merits and demerits. Based on these, we propose a novel multiuser beamforming scheme to flexibly combine the merits of the traditional beamforming schemes. In the final part of the paper, we give some simulation results to compare the beamforming schemes mentioned in the paper. These simulation results show the superiority of the proposed beamforming scheme.

\section{Introduction}

As of the exponential increasing load of wireless communications networks, research on network architecture and quality of service has been done in various fields [1-3]. As for communication systems, one of the most important problems is that the capacity of them becomes insufficient. In order to solve the problem, the concept of massive multiple input multiple output (MIMO), where the system employs unprecedented numbers of antennas to simultaneously serve dozens of user equipment (UE), was proposed [4].

A large number of surveys on massive MIMO have been done by communication researchers [5-8]. Both theoretical and measurement results indicate that massive MIMO is capable of achieving huge spectral efficiency by applying multiuser beamforming (MU-BF) schemes. The two most prominent beamforming schemes are maximum ratio transmission (MRT) and zero-forcing (ZF), which require the instantaneous channel state information at the transmitter (CSIT) [9-11].

Owing to the channel reciprocity in time-division duplex (TDD) massive MIMO systems, it is feasible to obtain the instantaneous CSIT, while the CSIT acquisition is a great challenge in frequency-division duplex (FDD) massive MIMO systems.
In FDD massive MIMO systems, the two largest problems are reference signal (RS) design and codebook design. A great deal of research has been done to solve the problems [12-14]. However, no matter RS design or codebook design, there exists an intractable problem: there has not been a good way for the BSs to simultaneously serve more than two UEs in the same time-frequency resources in FDD massive MIMO systems, as it is hard to select a subset of users due to high computation complexities with the large number of $3 \mathrm{D}$ codebooks.

In order to fully exploit the potential of massive MIMO in FDD systems, a beamforming (BF) scheme exploiting the spatial correlation (SC) was proposed [15]. Although SC-BF can simultaneously schedule tens of UEs, the system performance is poor. To further boost the system performance of $\mathrm{SC}-\mathrm{BF}$, we proposed a novel BF scheme based on both the SC and signal leakage (SCSL), which can balance the system performance and overhead of reference signal (RS) [16]. However, the main idea of SCSL-BF is to maximize the signalleakage-to-noise ratio, but when the scheduled users are large, the main factor that restricts the performance of massive MIMO system is the interference among scheduled users. Therefore, the system performance gain of SCSL-BF is not large enough. In addition, the calculation of SCSL requires 
the whole-dimension channel matrix; thus, the calculation complexity of it is very high.

In this paper, we consider that the BSs feed back quantized-channel-codebook index. Based on the quantized channel, we put forward a SC and interference suppression (SCIS) based MU-BF scheme, to make it feasible for the BSs to simultaneously schedule tens of UEs in FDD massive MIMO systems. The proposed BF scheme only needs partial CSIT, which can be derived by transmitting some affordable RSs. Therefore, the RS overhead in FDD massive MIMO systems is flexible. With the partial CSIT, SCIS-BF can be transformed from SC-BF by a transformation matrix and a rotation angle. The transformation matrix can be deduced through Householder rotation, and the rotation angle can be derived by transmitting a RS in the downlink. Moreover, based on the quantized-channel-codebook and quantizedchannel-selection approach given in this paper, we evaluate the performance of the proposed SCIS-BF scheme with different levels of feedback overhead, and numerical results indicate that SCIS-BF performs better than SC-BF when UEs only feed back a few bits of quantized-channel-codebook index.

The remainder of the paper is organized as follows. Section 2 describes the system model. The details of the proposed SCIS-BF scheme are presented in Section 3. Section 4 evaluates the performance of SCIS-BF and Section 5 concludes this paper.

Notations. We use boldface capital letters $\mathbf{X}$ for matrices, boldface small letters $\mathbf{x}$ for vectors, and small letters $x$ for scalars. $\mathbb{E}\{\cdot\}$ is the expectation operator and $\mathbb{C}$ and $\mathbb{N}$ denote the set of real and complex numbers with specified dimensions. $[\cdot]^{\mathrm{T}},[\cdot]^{\mathrm{H}}$, and $[\cdot]^{-1}$ denote the transpose, the Hermitian transpose, and the inverse, respectively. $\mathbf{I}_{N}$ represents an identity matrix of size $N$, and $\mathbf{x} \sim \mathbb{C N}(\mathbf{u}, \mathbf{R})$ indicates that $\mathbf{x}$ is a circularly symmetric complex Gaussian vector with mean $\mathbf{u}$ and covariance matrix $\mathbf{R}$.

\section{System Model}

As depicted in Figure 1, this paper focuses on FDD massive MIMO systems, where the BSs communicate with $K$ single-antenna users simultaneously. The users in each cell receive the signal transmitted from BS of the cell, and at the same time, they suffer from both the intercell interference and the intracell interference. Figure 2 is the transmission model of each BS. As Figure 2 shows, in each cell, the BS transmits RSs in the downlink; then with the received signals, the users estimate the channels and feed back a $L$-bit quantized-channel-codebook index in the uplink.

2.1. 3D Antenna Modeling. In this part, we mainly introduce the antenna modeling of the 3D channel used in this paper [17]. In the 3D channel model, 2D planar antenna array is considered. As to the planar antenna array, the spatial correlation is affected by the radiation pattern as well as the distance of antenna elements, regardless of the electromagnetic coupling between antenna elements and the effects of

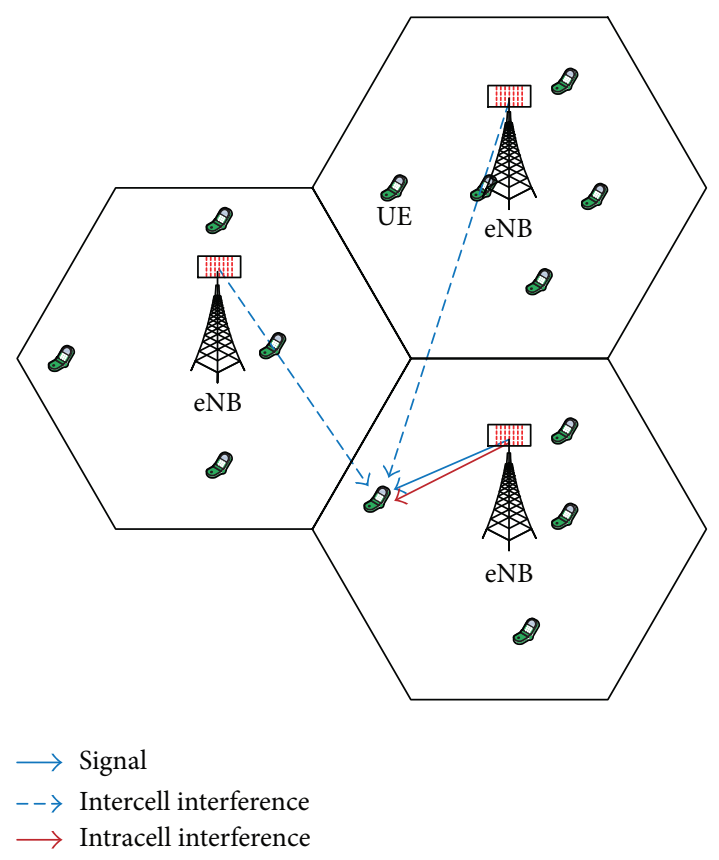

Figure 1: System model.

impedance mismatch. As depicted in Figure 3, the directional antenna elements radiation pattern is denoted as [18]

$$
\begin{aligned}
& A(\phi, \theta)=-\min \left\{-\left[A_{E, H}(\phi)+A_{E, V}(\theta)\right], A_{m}\right\}, \\
& A_{E, H}(\phi)=-\min \left[12\left(\frac{\phi}{\phi_{3 \mathrm{~dB}}}\right)^{2}, A_{m}\right] \\
& A_{E, V}(\theta)=-\min \left[12\left(\frac{\theta-90^{\circ}}{\theta_{3 \mathrm{~dB}}}\right)^{2}, \mathrm{SLA}_{V}\right]
\end{aligned}
$$

where $\phi$ and $\theta$ are azimuth angle and zenith angle. $A_{E, H}(\phi)$ and $A_{E, V}(\theta)$ are the horizontal pattern and elevation pattern antenna gain, and $A_{m}$ and $\mathrm{SLA}_{V}$ denote the maximum attenuation and the slide lobe attenuation in vertical direction, respectively.

2.2. Channel Model. In this paper, we select the 3GPP 3D channel as the reference channel [17]. Denote $\mathbf{h}_{k}=$ $\widetilde{\mathbf{h}}_{k} \mathbf{R}_{k}^{1 / 2} \in \mathbb{C}^{1 \times N}$ as the channel vector from the BS to user $k$, where $\widetilde{\mathbf{h}}_{k} \sim \mathbb{C N}\left(0, \mathbf{I}_{N}\right)$ contains independent and identically distributed (i.i.d.) elements and $\mathbf{R}_{k}$ represents the transmit correlation matrix. The elements of $\mathbf{R}_{k}$, namely, $\left[\mathbf{R}_{k}\right]_{p q} \forall p, q \in\{1,2, \ldots, N\}$, are defined as

$$
\left[\mathbf{R}_{k}\right]_{p q}=\frac{\mathbb{E}\left\{\left[\mathbf{h}_{k}\right]_{p}^{\mathrm{H}}\left[\mathbf{h}_{k}\right]_{q}\right\}}{\sqrt{\mathbb{E}\left\{\left[\mathbf{h}_{k}\right]_{p}^{\mathrm{H}}\left[\mathbf{h}_{k}\right]_{p}\right\} \mathbb{E}\left\{\left[\mathbf{h}_{k}\right]_{q}^{\mathrm{H}}\left[\mathbf{h}_{k}\right]_{q}\right\}}} .
$$

In terms of $3 \mathrm{D}$ beamforming steering direction, the transmit correlation matrix can also be defined as

$$
\left[\mathbf{R}_{k}\right]_{p q}=\frac{\left[\mathbf{R}_{\mathrm{C}}\right]_{p q}}{\sqrt{\left[\mathbf{R}_{\mathrm{C}}\right]_{p p}\left[\mathbf{R}_{\mathrm{C}}\right]_{q q}}},
$$




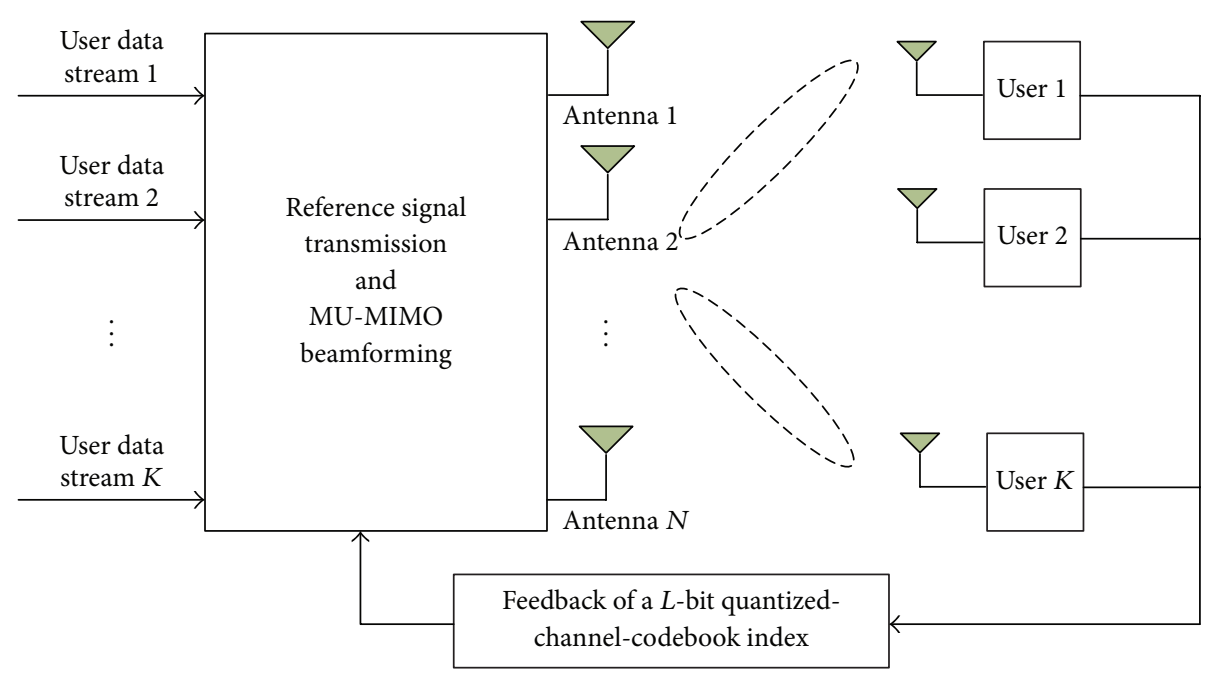

Figure 2: Transmission model.

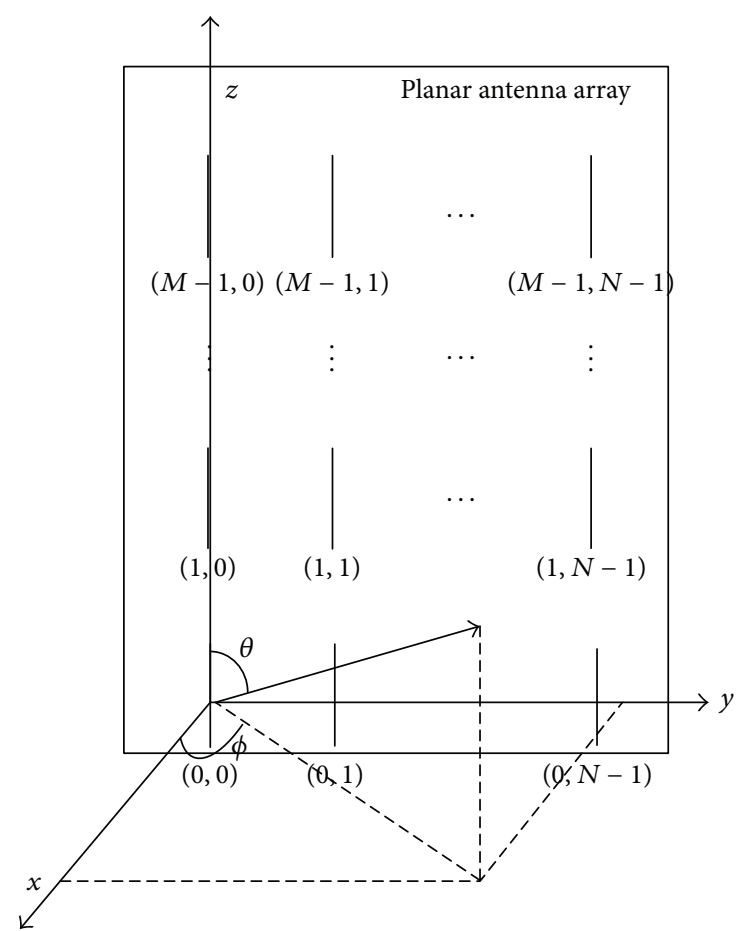

Figure 3: 3D antenna model.

where $\mathbf{R}_{C}$ is the transmit covariance matrix, which is given by

$$
\mathbf{R}_{\mathrm{C}}=\oiint \alpha^{\mathrm{H}}(\phi, \theta) \alpha(\phi, \theta) P(\phi, \theta) A(\phi, \theta) d \phi d \theta .
$$

In (4), $\alpha(\phi, \theta)$ is the $3 \mathrm{D}$ steering row vector, $P(\phi, \theta)$ is the power angular spread (PAS) of 3D channel, and $A(\phi, \theta)$ is the aforementioned antenna pattern transformed to linear scale.

\subsection{Channel Quantization}

2.3.1. DFT-Based Codebook. In this part, we talk about the channel quantization. A simple way to design the quantized-channel codebook is based on DFT. Suppose that the BSs select $n$ antennas to transmit RSs and the number of quantized-channel vectors in the codebook is $N_{c}=2^{L}$, where $L$ denotes the number of feedback bits of codebook index. Then the quantized-channel vector $\widehat{\mathbf{h}}_{m}$ based on DFT can be expressed as

$$
\widehat{\mathbf{h}}_{m}=\frac{1}{\sqrt{n}}\left[1, \mathrm{e}^{j 2 \pi m / N_{c}}, \ldots, \mathrm{e}^{\mathrm{j} 2 \pi m(n-1) / N_{c}}\right]
$$

where $m \in\left\{1,2, \ldots, N_{c}\right\}$ denotes the quantized-channel vector index in the codebook.

2.3.2. Quantized-Channel-Vector Selection. To select the quantized-channel vector from the above-mentioned codebook, we firstly define $\overline{\mathbf{h}}_{k} \in \mathbb{C}^{1 \times n}$ as the partial channels of user $k$ and $\widehat{\mathbf{h}}$ as the quantized-channel vector of $\overline{\mathbf{h}}_{k}$. Then the quantized error vector $\widehat{\boldsymbol{\varepsilon}}_{k}$ can be expressed as

$$
\widehat{\boldsymbol{\varepsilon}}_{k}=\overline{\mathbf{h}}_{k}-\left\|\overline{\mathbf{h}}_{k}\right\|_{2} \widehat{\mathbf{h}} .
$$

The criterion of quantized-channel-vector selection is to find $\widehat{\mathbf{h}}$ to minimize $\left|\widehat{\boldsymbol{\varepsilon}}_{k} \widehat{\boldsymbol{\varepsilon}}_{k}^{\mathrm{H}}\right|$. That is to say, select the quantizedchannel vector $\widehat{\mathbf{h}}$ such that

$$
\widehat{\mathbf{h}}=\arg \max _{m}\left|\overline{\mathbf{h}}_{k} \widehat{\mathbf{h}}_{m}^{\mathrm{H}}\right| .
$$

2.4. Downlink Transmission Model. Suppose $\mathbf{x} \in \mathbb{C}^{K \times 1}$ as the transmitted symbol vector with $\mathbb{E}\left[\mathbf{x x}^{\mathrm{H}}\right]=\mathbf{I}_{K}$ and $\mathbf{W}=$ $\left[\mathbf{w}_{1}, \mathbf{w}_{2}, \ldots, \mathbf{w}_{K}\right] \in \mathbb{C}^{N \times K}$ as the beamforming matrix with $\left\|\mathbf{w}_{k}\right\|_{2}=1(k=1,2, \ldots, K)$; then the downlink transmission model of user $k$ can be expressed as

$$
y_{k}=\mathbf{h}_{k} \mathbf{W} \mathbf{x}+n_{k}=\mathbf{h}_{k} \mathbf{w}_{k} x_{k}+i_{k}+n_{k},
$$

where $n_{k} \sim \mathbb{C N}\left(0, \sigma^{2}\right)$ is the additive white Gaussian noise and $i_{k}$ denotes the interferences from other users. 
Based on the aforementioned channel model, the received signal of user $k$ can be rewritten as

$$
y_{k}=\widetilde{\mathbf{h}}_{k} \mathbf{R}_{k}^{1 / 2} \mathbf{w}_{k} x_{k}+i_{k}+n_{k} .
$$

\section{BF Schemes in Massive MIMO Systems}

In this section, we first review two conventional BF schemes, namely, ZF-BF scheme and SC-BF scheme. Then, a novel BF scheme exploiting both the SC matrix and partial channels is proposed to synthesize the merits of the two types of $\mathrm{BF}$ schemes.

\subsection{Conventional Beamforming Schemes}

3.1.1. Zero-Forcing Beamforming. The criterion of ZF-BF is to eliminate the interferences among users. Define $\mathbf{W}_{\mathrm{ZF}}=$ $\left[\mathbf{w}_{1}, \mathbf{w}_{2}, \ldots, \mathbf{w}_{K}\right] \in \mathbb{C}^{N \times K}$ as the $\mathrm{ZF}$ beamforming matrix and $\mathbf{H}=\left[\mathbf{h}_{1}^{\mathrm{T}}, \mathbf{h}_{2}^{\mathrm{T}}, \ldots, \mathbf{h}_{K}^{\mathrm{T}}\right]^{\mathrm{T}} \in \mathbb{C}^{K \times N}$ as the channel matrix; then $\mathbf{W}_{\mathrm{ZF}}$ can be expressed as

$$
\mathbf{W}_{\mathrm{ZF}}=\mathbf{H}^{\mathrm{H}}\left(\mathbf{H} \mathbf{H}^{\mathrm{H}}\right)^{-1}
$$

3.1.2. Spatial Correlation Beamforming. As for SC-BF, the criterion is to maximize the signal power of the desired user by exploiting the spatial correlation without considering the interference to other users. From [19], we know that the weight vector of SC-BF of user $k$ can be expressed as

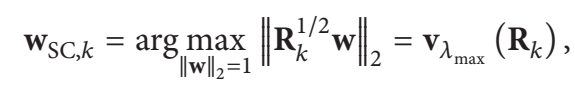

where $\mathbf{v}_{\lambda_{\max }}\left(\mathbf{R}_{k}\right)$ denotes the eigenvector corresponding to the largest eigenvalue $\lambda_{\max }$ of $\mathbf{R}_{k}$.

Compared to ZF-BF, SC-BF only needs the quasi-static SC matrix, which can significantly simplify the BF process and release the dependence on the CSIT in LS-MIMO systems. Nevertheless, the system performance degrades correspondingly.

3.2. Proposed Beamforming Scheme. Ignoring the subscript of user index, notice that $\left\|\mathbf{w}_{\mathrm{ZF}}\right\|_{2}=\left\|\mathbf{w}_{\mathrm{SC}}\right\|_{2}=1$, and we can construct a unitary transformation $\mathbf{G}$ to transform $\mathbf{w}_{\mathrm{SC}}$ and make it close to $\mathbf{w}_{\mathrm{ZF}}$ in order to improve the system by exploiting partial channels.

That is to say, the proposed SCIS-BF weight vector $\mathbf{w}_{\mathrm{SCIS}}$ satisfies

$$
\mathbf{w}_{\text {SCIS }}=\mathbf{G}_{\mathbf{h}} \mathbf{w}_{\mathrm{SC}} \stackrel{\overline{\mathbf{h}}=\mathbf{h}}{=} \mathrm{e}^{j \omega} \widehat{\mathbf{w}}_{\mathrm{ZF}},
$$

where $\mathrm{e}^{j \omega} \widehat{\mathbf{w}}_{\mathrm{ZF}}$ denotes a linear correlated vector of $\widehat{\mathbf{w}}_{\mathrm{ZF}}$ and $\overline{\mathbf{h}}$ is a part of the channel vector $\mathbf{h}$, given by

$$
[\overline{\mathbf{h}}]_{i}=[\mathbf{h}]_{N_{i}}
$$

with $i \in\{1,2, \ldots, n\}$. We define $\mathbb{N}=\left\{N_{1}, N_{2}, \ldots, N_{n}\right\} \subset$ $\{1,2, \ldots, N\}$ as the index set of the selected $n$ antennas. Therefore, we can construct the SCIS-BF weight vector by deducing the transform matrix $\mathbf{G}_{\overline{\mathbf{h}}}$ and rotation angle $\omega$, respectively.

Next, with the quantized-channel vector fed back in the uplink, we will deduce transform matrix $\mathbf{G}_{\overline{\mathbf{h}}}$ and rotation angle $\omega$ for a fixed index set $\mathbb{N}$, respectively.

3.2.1. Derivation of Transform Matrix. There exists a unitary transformation matrix $\mathbf{G}_{\overline{\mathbf{h}}}$ such that

$$
\mathbf{w}_{\text {SCIS }}=\mathbf{G}_{\overline{\mathbf{h}}} \mathbf{w}_{\mathrm{SC}}\left\{\begin{array}{l}
\stackrel{\mathbb{N}=\emptyset}{=} \mathbf{w}_{\mathrm{SC}}, \\
\stackrel{\mathbb{N}=\{1,2, \ldots, N\}}{=} \mathrm{e}^{j \omega} \widehat{\mathbf{w}}_{\mathrm{ZF}} .
\end{array}\right.
$$

Proof. Denote $\widehat{\mathbf{H}}=\left[\widehat{\mathbf{h}}_{1}^{\mathrm{T}}, \widehat{\mathbf{h}}_{2}^{\mathrm{T}}, \ldots, \widehat{\mathbf{h}}_{K}^{\mathrm{T}}\right]^{\mathrm{T}}$ as the quantizedchannel matrix of $\overline{\mathbf{H}}$; then the ZF beamforming matrix with quantized partial channels can be expressed as [20]

$$
\widehat{\mathbf{W}}_{\mathrm{ZF}}=\widehat{\mathbf{H}}^{\mathrm{H}}\left(\widehat{\mathbf{H}} \widehat{\mathbf{H}}^{\mathrm{H}}\right)^{-1}
$$

where $\widehat{\mathbf{W}}_{\mathrm{ZF}}=\left[\widehat{\mathbf{w}}_{\mathrm{ZF}, 1}, \widehat{\mathbf{w}}_{\mathrm{ZF}, 2}, \ldots, \widehat{\mathbf{w}}_{\mathrm{ZF}, K}\right]$ and $\widehat{\mathbf{w}}_{\mathrm{ZF}, k}$ denotes the $\mathrm{ZF}-\mathrm{BF}$ weight vector of user $k$ with quantized partial channels. For convenience, we ignore the subscript of user index.

In the unitary space $\mathbb{C}^{N \times 1}$, we assume

$$
[\mathbf{z}]_{i}= \begin{cases}{\left[\mathrm{e}^{j \omega} \widehat{\mathbf{w}}_{\mathrm{ZF}}\right]_{i},} & i \in\{1,2, \ldots, n\}, \\ 0, & \text { others, }\end{cases}
$$

and define

$$
\left[\overline{\mathbf{w}}_{\mathrm{SC}}\right]_{i}= \begin{cases}{\left[\mathbf{w}_{\mathrm{SC}}\right]_{N_{i}},} & i \in\{1,2, \ldots, n\}, \\ 0, & \text { others }\end{cases}
$$

and then resorting to Householder rotation, we can find two unitary transform matrixes $\mathbf{G}_{1}$ and $\mathbf{G}_{2}$ such that

$$
\begin{gathered}
\mathbf{G}_{1} \mathbf{z}=\|\mathbf{z}\|_{2} \mathrm{e}^{j(\pi / 4)} \mathbf{e}_{1}, \\
\mathbf{G}_{2} \overline{\mathbf{w}}_{\mathrm{SC}}=\left\|\overline{\mathbf{w}}_{\mathrm{SC}}\right\|_{2} \mathrm{e}^{j(\pi / 4)} \mathbf{e}_{1},
\end{gathered}
$$

where $\mathbf{e}_{1}$ is the unit base vector with the first component equal to 1 . The proof is as follows.

Assume $\mathbf{x} \in \mathbb{C}^{N \times 1}$ and we define $a=\|\mathbf{x}\|_{2} \mathrm{e}^{j(\pi / 4)}$ and $\mathbf{G}(\mathbf{w})=\left(\mathbf{I}_{N}-2 \mathbf{w} \mathbf{w}^{\mathrm{H}}\right)$, where

$$
\mathbf{w}=\frac{\mathbf{x}-a \mathbf{e}_{1}}{\left\|\mathbf{x}-a \mathbf{e}_{1}\right\|_{2}} .
$$

Then we can obtain

$$
\begin{aligned}
\mathbf{G}(\mathbf{w}) \mathbf{x} & =\left(\mathbf{I}_{N}-2 \frac{\left(\mathbf{x}-a \mathbf{e}_{1}\right)\left(\mathbf{x}-a \mathbf{e}_{1}\right)^{\mathrm{H}}}{\left\|\mathbf{x}-a \mathbf{e}_{1}\right\|_{2}^{2}}\right) \mathbf{x} \\
& =\mathbf{x}-2 \frac{\left(\mathbf{x}-a \mathbf{e}_{1}\right)\left(\mathbf{x}-a \mathbf{e}_{1}\right)^{\mathrm{H}} \mathbf{x}}{\left(\mathbf{x}-a \mathbf{e}_{1}\right)^{\mathrm{H}}\left(\mathbf{x}-a \mathbf{e}_{1}\right)},
\end{aligned}
$$


where $\left(\mathbf{x}-a \mathbf{e}_{1}\right)^{\mathrm{H}}\left(\mathbf{x}-a \mathbf{e}_{1}\right)$ meets

$$
\begin{aligned}
\left(\mathbf{x}-a \mathbf{e}_{1}\right)^{\mathrm{H}}\left(\mathbf{x}-a \mathbf{e}_{1}\right)= & \mathbf{x}^{\mathrm{H}} \mathbf{x}-a \mathbf{x}^{\mathrm{H}} \mathbf{e}_{1}-\bar{a} \mathbf{e}_{1}^{\mathrm{H}} \mathbf{x}+|a|^{2} \\
= & \mathbf{x}^{\mathrm{H}} \mathbf{x}-\left(a \mathbf{x}^{\mathrm{H}} \mathbf{e}_{1}\right)^{\mathrm{H}}-\bar{a} \mathbf{e}_{1}^{\mathrm{H}} \mathbf{x} \\
& +\mathbf{x}^{\mathrm{H}} \mathbf{x}=2\left(\mathbf{x}^{\mathrm{H}} \mathbf{x}-\bar{a} \mathbf{e}_{1}^{\mathrm{H}} \mathbf{x}\right) \\
= & 2\left(\mathbf{x}-a \mathbf{e}_{1}\right)^{\mathrm{H}} \mathbf{x} .
\end{aligned}
$$

Finally, we can obtain

$$
\begin{aligned}
\mathbf{G}(\mathbf{w}) \mathbf{x} & =\mathbf{x}-2 \frac{\left(\mathbf{x}-a \mathbf{e}_{1}\right)\left(\mathbf{x}-a \mathbf{e}_{1}\right)^{\mathrm{H}} \mathbf{x}}{\left(\mathbf{x}-a \mathbf{e}_{1}\right)^{\mathrm{H}}\left(\mathbf{x}-a \mathbf{e}_{1}\right)} \\
& =\mathbf{x}-2 \frac{\left(\mathbf{x}-a \mathbf{e}_{1}\right)\left(\mathbf{x}-a \mathbf{e}_{1}\right)^{\mathrm{H}} \mathbf{x}}{2\left(\mathbf{x}-a \mathbf{e}_{1}\right)^{\mathrm{H}} \mathbf{x}}=a \mathbf{e}_{1} .
\end{aligned}
$$

From the above proof we can derive that $\mathbf{G}_{1} \mathbf{z} /\|\mathbf{z}\|_{2} \mathrm{e}^{j(\pi / 4)}=$ $\mathbf{G}_{2} \overline{\mathbf{w}}_{\mathrm{SC}} /\left\|\overline{\mathbf{w}}_{\mathrm{SC}}\right\|_{2} \mathrm{e}^{j(\pi / 4)}=\mathbf{e}_{1}$. Define $\mathbf{G}_{\overline{\mathbf{h}}}=\left[\mathbf{G}_{1}\right]^{-1} \mathbf{G}_{2}$ and it satisfies the fact that

$$
\mathbf{G}_{\mathbf{h}} \overline{\mathbf{w}}_{\mathrm{SC}}=\left[\mathbf{G}_{1}\right]^{-1} \mathbf{G}_{2} \overline{\mathbf{w}}_{\mathrm{SC}}=\frac{\left\|\overline{\mathbf{w}}_{\mathrm{SC}}\right\|_{2}}{\|\mathbf{z}\|_{2}} \mathbf{z} .
$$

Let $\mathbf{w}_{\mathrm{SCIS}}=\mathbf{G}_{\overline{\mathbf{h}}} \mathbf{w}_{\mathrm{SC}}$, and we can arrive at

$$
\left[\mathbf{w}_{\mathrm{SCIS}}\right]_{N_{i}}= \begin{cases}{\left[\mathbf{G}_{\overline{\mathbf{h}}} \overline{\mathbf{w}}_{\mathrm{SC}}\right]_{i},} & N_{i} \in \mathbb{N}, \\ {\left[\mathbf{w}_{\mathrm{SC}}\right]_{N_{i}},} & \text { others. }\end{cases}
$$

Therefore, with the quantized partial channels, we can finally obtain

$$
\mathbf{w}_{\text {SCIS }} \stackrel{\mathbb{N}=\emptyset}{=} \mathbf{w}_{\text {SC }} \stackrel{\mathbb{N}=\{1,2, \ldots, N\}}{=} \mathrm{e}^{j \omega} \widehat{\mathbf{w}}_{\mathrm{ZF}}
$$

Here, we have to note that the ZF-BF weight vector is calculated from reduced-dimension channel matrix; thus, the calculation complexity is adjustable and depends on the antenna-selection proportion. Moreover, it is easy to obtain the transformation matrix. As to SCSL-BF, the SL$\mathrm{BF}$ weight vector is calculated from the whole-dimension, no matter what the antenna-selection proportion. Besides, the transformation matrix of SCSL-BF needs many times of matrix multiplication [13]. Therefore, compared to SCSL-BF, the complexity to calculate SCIS-BF weight vector is relatively low.

3.2.2. Calculation of Rotation Angle. The rotation angle in expression (7) is given by

$$
\omega=\angle\left(\mathbf{h w}_{\mathrm{SC}}-\widehat{\mathbf{h}}_{s} \overline{\mathbf{w}}_{\mathrm{SC}}\right)-\angle\left(\widehat{\mathbf{h}} \widehat{\mathbf{w}}_{\mathrm{ZF}}\right) .
$$

Proof. The calculation of the rotation angle $\omega$ needs a careful consideration. From the above analysis, define $\widehat{\mathbf{h}} \widehat{\mathbf{w}}_{\mathrm{ZF}}=r \mathrm{e}^{j \theta}$ and

$$
\left[\widehat{\mathbf{h}}_{s}\right]_{i}= \begin{cases}{[\widehat{\mathbf{h}}]_{i},} & i \in\{1,2, \ldots, n\}, \\ 0, & \text { others }\end{cases}
$$

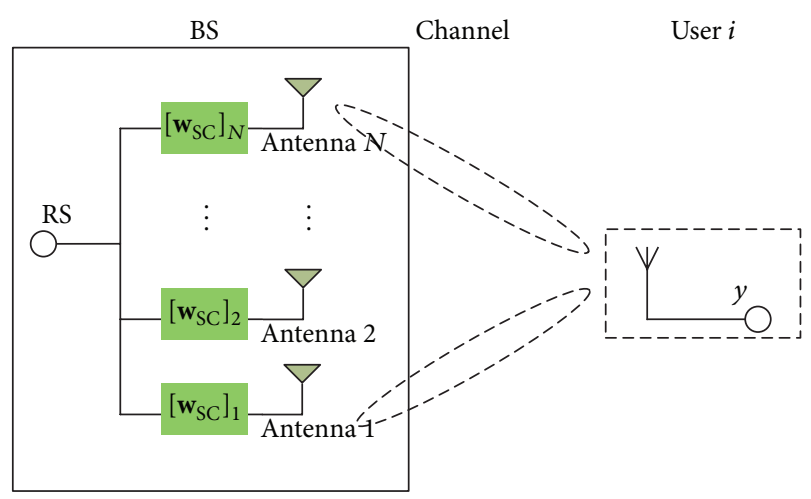

FiguRE 4: RS transmission model to get $\mathbf{h} \mathbf{w}_{\mathrm{SC}}$.

and we can get the following relation:

$$
\begin{aligned}
\mathbf{h w}_{\mathrm{SCIS}} & =\sum_{i=1}^{N} \mathbf{h}_{i}\left[\mathbf{w}_{\mathrm{SCIS}}\right]_{i} \\
& =\sum_{i \in \mathbb{N}} \mathbf{h}_{i}\left[\mathbf{w}_{\mathrm{SCIS}}\right]_{i}+\sum_{i \notin \mathbb{N}} \mathbf{h}_{i}\left[\mathbf{w}_{\mathrm{SCIS}}\right]_{i} \\
& \approx \frac{\left\|\overline{\mathbf{w}}_{\mathrm{SC}}\right\|_{2}}{\|\mathbf{z}\|_{2}} e^{j \omega} \widehat{\mathbf{h}}_{s} \mathbf{z}+\mathbf{h} \mathbf{w}_{\mathrm{SC}}-\widehat{\mathbf{h}}_{s} \overline{\mathbf{w}}_{\mathrm{SC}} \\
& =\frac{\left\|\overline{\mathbf{w}}_{\mathrm{SC}}\right\|_{2}}{\|\mathbf{z}\|_{2}} \widehat{\mathbf{h}}_{\mathrm{ZF}} e^{j \omega}+\mathbf{h w}_{\mathrm{SC}}-\widehat{\mathbf{h}}_{s} \overline{\mathbf{w}}_{\mathrm{SC}} \\
& =\frac{\left\|\overline{\mathbf{w}}_{\mathrm{SC}}\right\|_{2}}{\|\mathbf{z}\|_{2}} r e^{j \theta} e^{j \omega}+\mathbf{h} \mathbf{w}_{\mathrm{SC}}-\widehat{\mathbf{h}}_{s} \overline{\mathbf{w}}_{\mathrm{SC}} .
\end{aligned}
$$

In order to maximize $\left|\mathbf{h} \mathbf{w}_{\mathrm{SCIS}}\right|$, the rotation angle $\omega$ should meet

$$
\omega=L\left(\mathbf{h w}_{\mathrm{SC}}-\widehat{\mathbf{h}}_{s} \overline{\mathbf{w}}_{\mathrm{SC}}\right)-\theta .
$$

Expression (29) indicates that we have to calculate $\mathbf{h w}_{\mathrm{SC}}$, $\widehat{\mathbf{h}}_{s} \overline{\mathbf{w}}_{\mathrm{SC}}$, and $\widehat{\mathbf{h}} \widehat{\mathbf{w}}_{\mathrm{ZF}}$ first in order to calculate the rotation angle. $\widehat{\mathbf{h}}$ is the quantized partial channel vector, which can be got by transmitting the affordable RSs and $\widehat{\mathbf{h}}_{s}$ can be calculated from expression (27). Besides, we can calculate $\widehat{\mathbf{w}}_{\mathrm{ZF}}$ and $\overline{\mathbf{w}}_{\mathrm{SC}}$ according to expressions (15) and (17), respectively. Therefore, we only need to compute $\mathbf{h w}_{\mathrm{SC}}$.

Although the BS is ignorant of the full channel vector h, it can transmit a RS mapped by the SC-BF weight vector $\mathbf{w}_{\mathrm{SC}}$ on each antenna, as depicted in Figure 4 . At the receiver, the UEs can receive a symbol containing the product of $\mathbf{w}_{\mathrm{SC}}$ and the RS. After that, the UEs can feed back $\mathbf{h w}_{\mathrm{SC}}$ to the BS. Therefore, we can finally get $\mathbf{h w}_{\mathrm{SC}}, \widehat{\mathbf{h}}_{s} \overline{\mathbf{w}}_{\mathrm{SC}}$, and $\widehat{\mathbf{h}} \widehat{\mathbf{w}}_{\mathrm{ZF}}$ for calculating the rotation angle $\omega$ while just taking one more RS in FDD LS-MIMO systems. Here, we have to note that the above $\mathbf{w}_{\mathrm{SC}}$ and $\mathbf{h} \mathbf{w}_{\mathrm{SC}}$ are all supposed to be ideally obtained. In the following sections, we also suppose that $\mathbf{w}_{\mathrm{SC}}$ can be ideally obtained. We do not talk about the qualification and feedback of it in this paper. 
TABLE 1: Parameters configuration.

\begin{tabular}{lc}
\hline Parameters & Settings \\
\hline Scenario & $3 \mathrm{D}-\mathrm{Uma}$ \\
Carrier frequency & $2 \mathrm{GHz}$ \\
Bandwidth & $5 \mathrm{MHz}$ \\
User speed & $3 \mathrm{~km} / \mathrm{h}$ \\
Channel model & 3GPP 3D channel model \\
eNB transmit power & $43 \mathrm{dBm}$ \\
Antenna gain & $8 \mathrm{dBi}$ \\
Antenna element pattern & BS: UPA (row $\times$ col: $12 \times 10)$ user: \\
Antenna element interval & single antenna \\
UE height & $0.5 \lambda$ \\
UE distribution & $1.5 \mathrm{~m}$ \\
Noise density & 30 UEs per sector, uniform in cell \\
Path loss/shadow fading/fast & $-174 \mathrm{dBm} / \mathrm{Hz}$ \\
fading & See TR36.873 [17] \\
\hline
\end{tabular}

\section{Evaluation}

\subsection{Simulation Setup}

4.1.1. Parameters Setting. To evaluate the proposed SCIS-BF scheme, we build a system-level simulation platform. In our simulation, the network model consists of 19 macrocells. Each cell contains 3 sectors, where each BS is located at the cell center with uniform planar array (UPA). The main simulation parameters are listed in Table 1. For the other relative channel parameters please refer to [13].

4.1.2. Antenna-Selection Approach. To evaluate the performance of SCIS-BF, we have to confirm the antenna index set firstly. From the above derivation, we can see that $\mathbb{N}$ can be an arbitrary subset of $\{1,2, \ldots, N\}$. Therefore, to simplify the problem in this paper, we give a simple approach to form antenna index set $\mathbb{N}$ as follows.

For antenna-selection approach $\left(\operatorname{SCIS}\left(n_{\mathrm{s}}\right)\right)$, select one antenna every $n_{\mathrm{s}}$ antennas to make the selected antennas distribute uniformly on the UPA.

4.2. Performance of SCIS-BF with Different Antenna-Selection Proportion. In this part, we evaluate the performance of SCIS-BF with different antenna-selection proportion. Fixing the number of scheduled users as 10, we compare the performance of SC, MRT, ZF, SCIS(2), SCIS(3), and SCIS(4).

Figure 5 shows the Cumulative Distribution Function (CDF) versus the signal-to-interference-and-noise ratio (SINR) of SCIS-BF with different antenna-selection proportion. Correspondingly, the CDF versus the UE throughput of SCIS-BF are shown in Figure 6. From the two figures, we can see that the performance of SCIS-BF is better than SC-BF, but worse than ZF-BF. When the antenna-selection proportion increases, SCIS-BF performs better and the performance of it approaches that of ZF-BF. It means that the proposed SCISBF can flexibly make a tradeoff between system performance

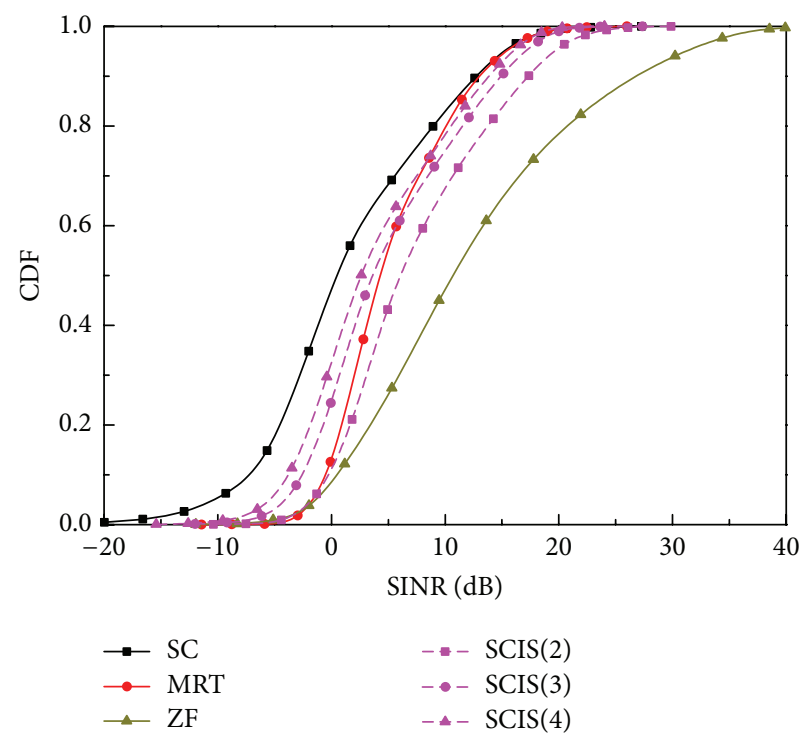

FIGURE 5: CDF versus SINR of SCIS-BF.

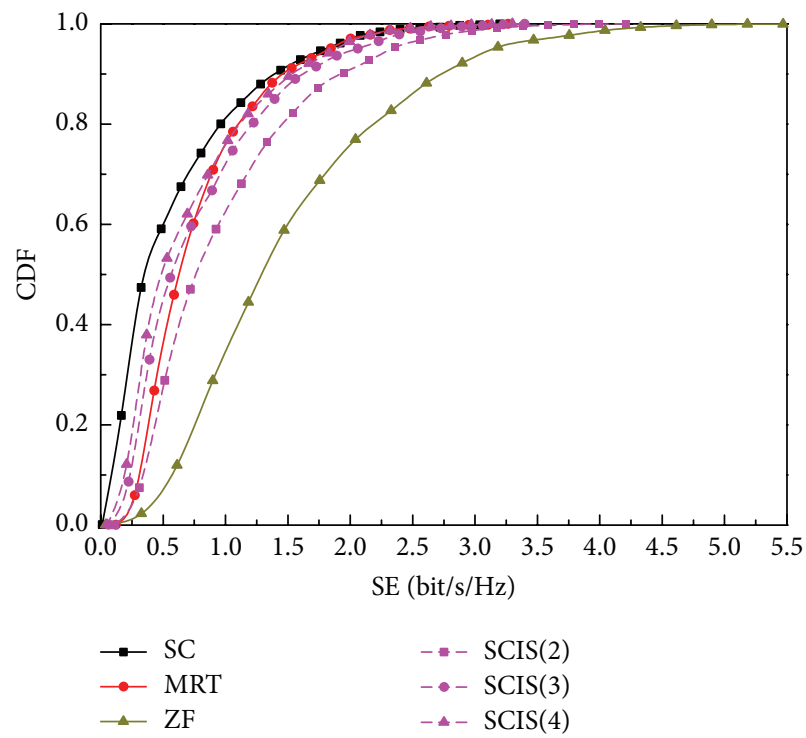

FIGURE 6: CDF versus throughput of SCIS-BF.

and RS overhead. In addition, when the antenna-selection proportion reaches a certain extent, SCIS-BF can outperform MRT.

4.3. Performance of SCIS-BF with Perfect CSIT. In this part, we evaluate the performance of SCIS-BF with perfect CSIT. With the antenna-selection approach given above, the spectral efficiency (SE) versus scheduled user numbers of different BF schemes is shown in Figure 7. From the figure, we can see that, as the number of scheduled users increases, the SE of all $\mathrm{BF}$ schemes increases correspondingly, and the performance of $\mathrm{ZF}-\mathrm{BF}$ is the best. However, the complexity of ZF-BF is very high and at the same time it needs the whole channel state information, which would cost great overhead of RSs in FDD massive MIMO systems. Besides, in Figure 7, both 
TABLE 2: Measurement of spectral efficiency.

\begin{tabular}{|c|c|c|c|c|c|c|}
\hline \multirow{2}{*}{$\begin{array}{l}\text { Feedback overhead } \\
\text { BF scheme }\end{array}$} & \multicolumn{2}{|c|}{$L=6$} & \multicolumn{2}{|c|}{$L=7$} & \multicolumn{2}{|c|}{$L=8$} \\
\hline & $\operatorname{SCSL}(3)$ & $\operatorname{SCIS}(3)$ & $\operatorname{SCSL}(3)$ & $\operatorname{SCIS}(3)$ & $\operatorname{SCSL}(3)$ & $\operatorname{SCIS}(3)$ \\
\hline System SE (bit/s/Hz) & 7.153 & 8.865 & 7.974 & 8.865 & 8.592 & 9.632 \\
\hline System SE gain (compared to SC) & $-9.73 \%$ & $11.88 \%$ & $0.63 \%$ & $19.40 \%$ & $8.43 \%$ & $21.55 \%$ \\
\hline
\end{tabular}

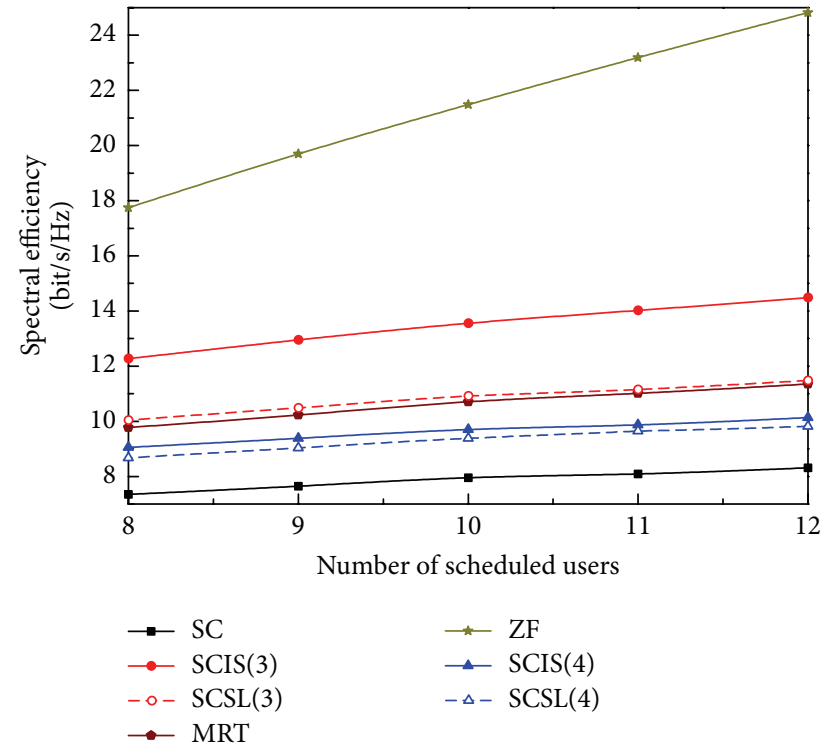

FIGURE 7: CDF versus SE of different BF schemes with perfect CSIT.

SCSL-BF and SCIS-BF perform better than SC-BF. When the numbers of selected antennas are the same, SCIS-BF outdoes SCSL-BF. Moreover, with the increasing number of selected antennas, SCIS-BF performs better, and, meanwhile, the difference between SCIS-BF and SCSL-BF becomes larger. It indicates that SCIS-BF is capable of balancing the system performance and overhead of RSs, and it has more potential than SCSL-BF.

4.4. Performance of SCIS-BF with Imperfect CSIT. Considering practical FDD systems, the BS does not know the real channel but the quantized-channel vector. Based on limited feedback, fixing the number of scheduled users as 10 , we evaluate the system performance of SCIS-BF in this part.

Figure 8 is the CDF versus SINR of SCIS-BF and SCSLBF with limited feedback, and Figure 9 shows the system SE of SCSL-BF and SCIS-BF under the condition of limited feedback. We can see that the performance of both SCSL and SCIS becomes better when gradually increasing the number of feedback bits. The corresponding SE measurements are listed in Table 2, where $L$ denotes the number of feedback bits. According to Table 2, we can see that implementing SCSL-BF in practical systems needs a large number of feedback bits, or the system performance would even be worse than SC-BF. Compared to SCSL-BF, it only needs a few feedback bits to implement SCIS-BF, and then the system performance could be improved. Therefore, to achieve

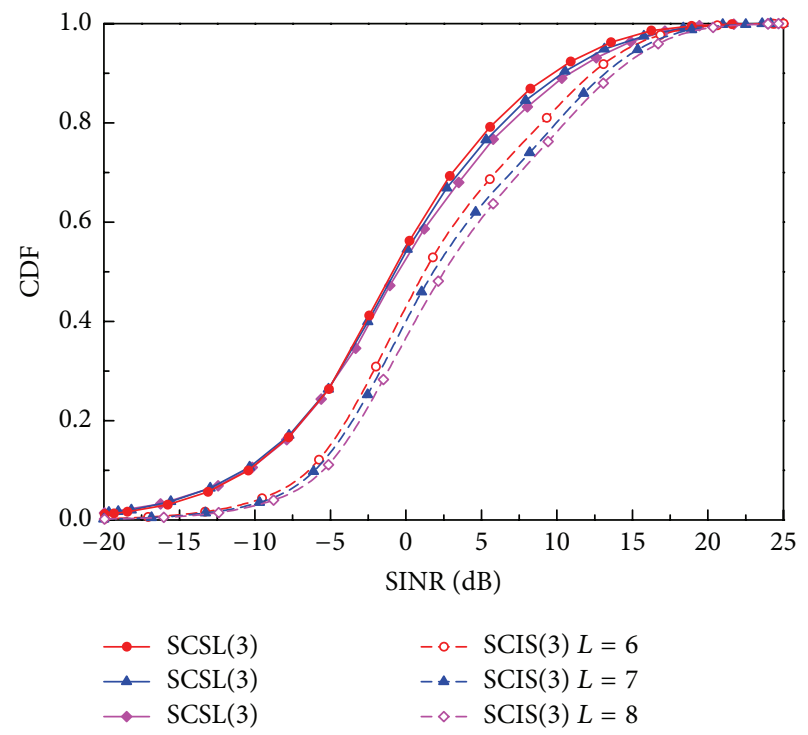

FIGURE 8: CDF versus SINR of SCSL and SCIS with imperfect CSIT.

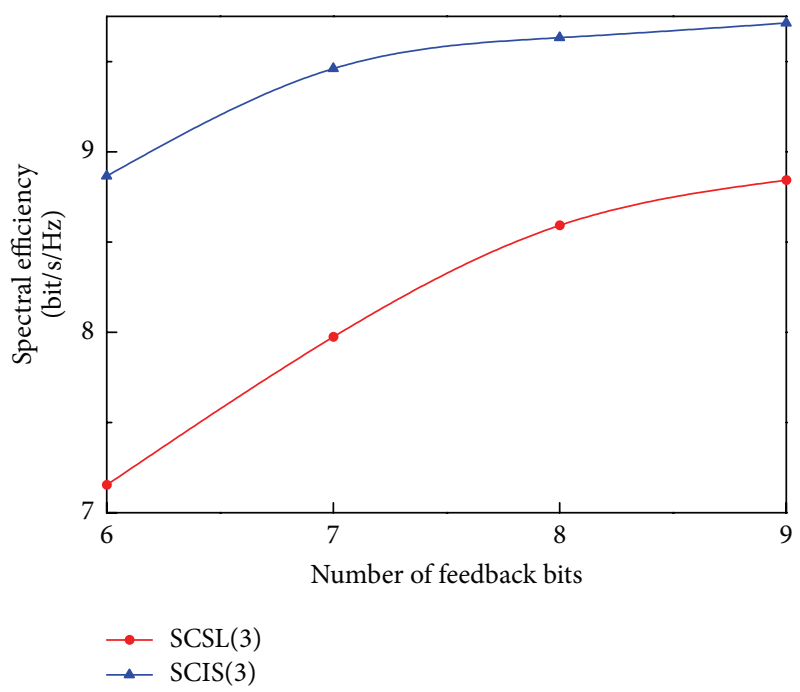

FIGURE 9: CDF versus SE of SCSL and SCIS with imperfect CSIT.

the same system performance, SCIS-BF needs less feedback overhead.

Table 3 summarizes the features of the mentioned BF schemes in this paper. It indicates that SCIS-BF could effectively balance the system performance and system overhead of both RSs and uplink feedback in practical systems. Therefore, SCIS-BF is a good candidate in FDD massive MIMO systems. 
TABLE 3: Feature of beamforming schemes.

\begin{tabular}{lcccccc}
\hline BF scheme & Spatial correlation & CSIT & Performance & RS overhead & Feedback overhead & Calculation complexity \\
\hline SC & $\sqrt{ }$ & $\times$ & Normal & Low & None & Low \\
\hline SCSL & $\sqrt{ }$ & $\sqrt{ }$ & Poor dynamic balance & High & High \\
\hline SCIS & $\sqrt{ }$ & $\sqrt{ }$ & Good dynamic balance & Low & Flexible \\
\hline
\end{tabular}

\section{Conclusion}

In this paper, we study the performance of different MU-BF schemes in massive MIMO systems, where the BSs feed back quantized-channel-codebook index, but not conventional precoding codebook index. Based on it, we put forward a novel SCIS-BF scheme in order to make it feasible for the BSs to simultaneously serve tens of UEs in the same timefrequency resources. In contrast to other conventional BF schemes, the calculation complexity of SCIS-BF is relatively low as it only requires the reduced-dimension channel matrix, and the performance of it is quite well. Besides, it can effectively balance the system performance and overhead of RSs. Furthermore, it only needs a few feedback bits to implement SCIS-BF in practical FDD massive MIMO systems. In a word, SCIS-BF can make it feasible and flexible to implement massive MIMO in FDD systems.

\section{Competing Interests}

The authors declare that there is no conflict of interests regarding the publication of this paper.

\section{Acknowledgments}

This work was supported by the National Key Scientific Instrument and Equipment Development Project under Grant 2013YQ20060706, the High Technology Research and Development Program of China under Grant 2014AA01A705, and the China Natural Science Funding (NSF) under Grant 61171106.

\section{References}

[1] K. Zheng, L. Hou, H. Meng et al., "Soft-defined heterogeneous vehicular network: architecture and challenges," http://arxiv .org/abs/1510.06579.

[2] K. Zheng, H. Meng, P. Chatzimisios, L. Lei, and X. Shen, "An SMDP-based resource allocation in vehicular cloud computing systems," IEEE Transactions on Industrial Electronics, vol. 62, no. 12, pp. 7920-7928, 2015.

[3] K. Zheng, X. Zhang, Q. Zheng, W. Xiang, and L. Hanzo, "Quality-of-experience assessment and its application to video services in LTE networks," IEEE Wireless Communications, vol. 22, no. 1, pp. 70-78, 2015.

[4] T. L. Marzetta, "Noncooperative cellular wireless with unlimited numbers of base station antennas," IEEE Transactions on Wireless Communications, vol. 9, no. 11, pp. 3590-3600, 2010.

[5] F. Rusek, D. Persson, B. K. Lau et al., "Scaling up MIMO: opportunities and challenges with very large arrays," IEEE Signal Processing Magazine, vol. 30, no. 1, pp. 40-60, 2013.
[6] K. Zheng, L. Zhao, J. Mei, B. Shao, W. Xiang, and L. Hanzo, "Survey of large-scale MIMO systems," IEEE Communications Surveys \& Tutorials, vol. 17, no. 3, pp. 1738-1760, 2015.

[7] K. Zheng, S. Ou, and X. Yin, "Massive MIMO channel models: a survey," International Journal of Antennas and Propagation, vol. 2014, Article ID 848071, 10 pages, 2014.

[8] H. Q. Ngo, E. G. Larsson, and T. L. Marzetta, "Energy and spectral efficiency of very large multiuser MIMO systems," IEEE Transactions on Communications, vol. 61, no. 4, pp. 1436-1449, 2013.

[9] H. Yang and T. L. Marzetta, "Performance of conjugate and zeroforcing beamforming in large-scale antenna systems," IEEE Journal on Selected Areas in Communications, vol. 31, no. 2, pp. 172-179, 2013.

[10] X. Gao, O. Edfors, F. Rusek, and F. Tufvesson, "Linear precoding performance in measured very-large MIMO channels," in Proceedings of the IEEE Vehicular Technology Conference (VTC Fall '11), pp. 1-5, IEEE, San Francisco, Calif, USA, September 2011.

[11] L. Zhao, K. Zheng, H. Long, and H. Zhao, "Performance analysis for downlink massive MIMO system with ZF precoding," Transactions on Emerging Telecommunications Technologies, vol. 25, no. 12, pp. 1219-1230, 2014.

[12] S. Wagner, R. Couillet, M. Debbah, and D. T. Slock, "Large system analysis of linear precoding in correlated MISO broadcast channels under limited feedback," IEEE Transactions on Information Theory, vol. 58, no. 7, pp. 4509-4537, 2012.

[13] Y. Song, S. Nagata, H. Jiang, and L. Chen, "CSI-RS design for 3D MIMO in future LTE-advanced," in Proceedings of the 1st IEEE International Conference on Communications (ICC '14), pp. 5101-5106, IEEE, Sydney, Australia, June 2014.

[14] Y. Xie, S. Jin, J. Wang, Y. Zhu, X. Gao, and Y. Huang, "A limited feedback scheme for 3D multiuser MIMO based on Kronecker product codebook," in Proceedings of the IEEE 24th Annual International Symposium on Personal, Indoor, and Mobile Radio Communications (PIMRC '13), pp. 1130-1135, London, UK, September 2013.

[15] A. Adhikary, J. Nam, J.-Y. Ahn, and G. Caire, "Joint spatial division and multiplexing - the large-scale array regime," IEEETransactions on Information Theory, vol. 59, no. 10, pp. 64416463, 2013.

[16] S. Zheng, H. Zhao, L. Zhao et al., "A novel beamforming scheme based on partial channel feedback in LS-MIMO systems," in Proceedings of the 10th EAI International Conference on Communications and Networking in China (Chinacom '15), Shanghai, China, August 2015.

[17] 3GPP, “3D channel model for LTE”, 3GPP TR 36.873 (V2.0.0), 2014.

[18] B. Shao, Y. Zhang, M. Xing, L. Zhao, and K. Zheng, "A novel beamforming scheme in FD-MIMO systems with spatial correlation," in Proceedings of the IEEE 25th International Symposium on Personal, Indoor and Mobile Radio Communications (PIMRC '14), pp. 313-317, Washington, DC, USA, September 2014. 
[19] J. Gutierrez, J. Ibanez, and J. Perez, "Spatial correlation beamforming scheme for MISO channel emulation," in Proceedings of the International Symposium on Wireless Communication Systems, pp. 446-450, Paris, France, August 2012.

[20] Z. Jianchi, L. Jingxiu, S. Xiaoming, and C. Lan, "Investigation on precoding techniques in E-UTRA and proposed adaptive precoding scheme for MIMO systems," in Proceedings of the 14th Asia-Pacific Conference on Communications (APCC '08), 5 pages, Tokyo, Japan, October 2008. 


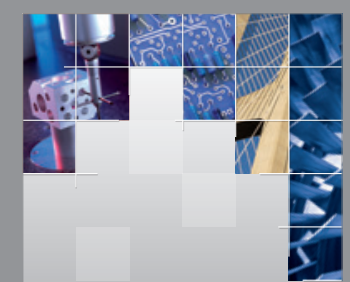

\section{Enfincering}
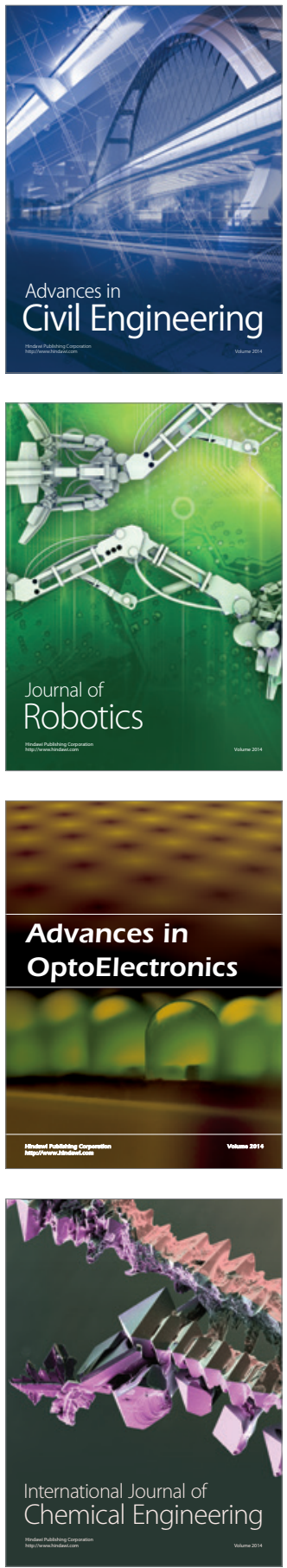

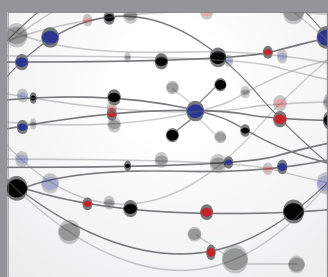

The Scientific World Journal

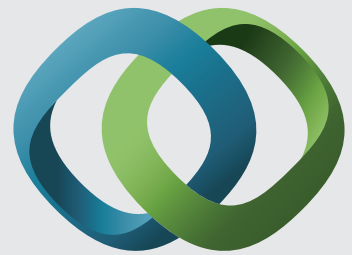

\section{Hindawi}

Submit your manuscripts at

http://www.hindawi.com
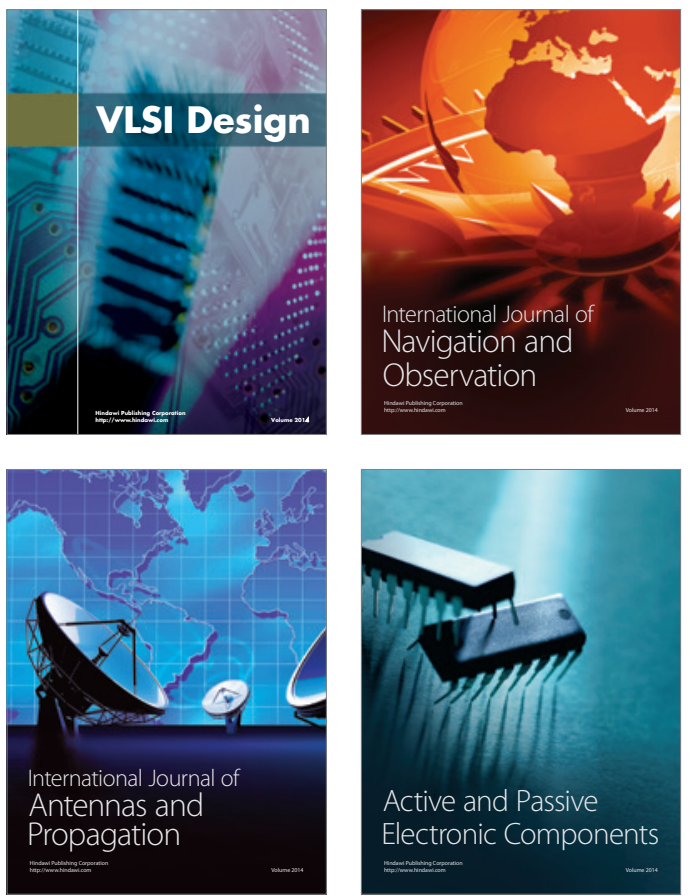
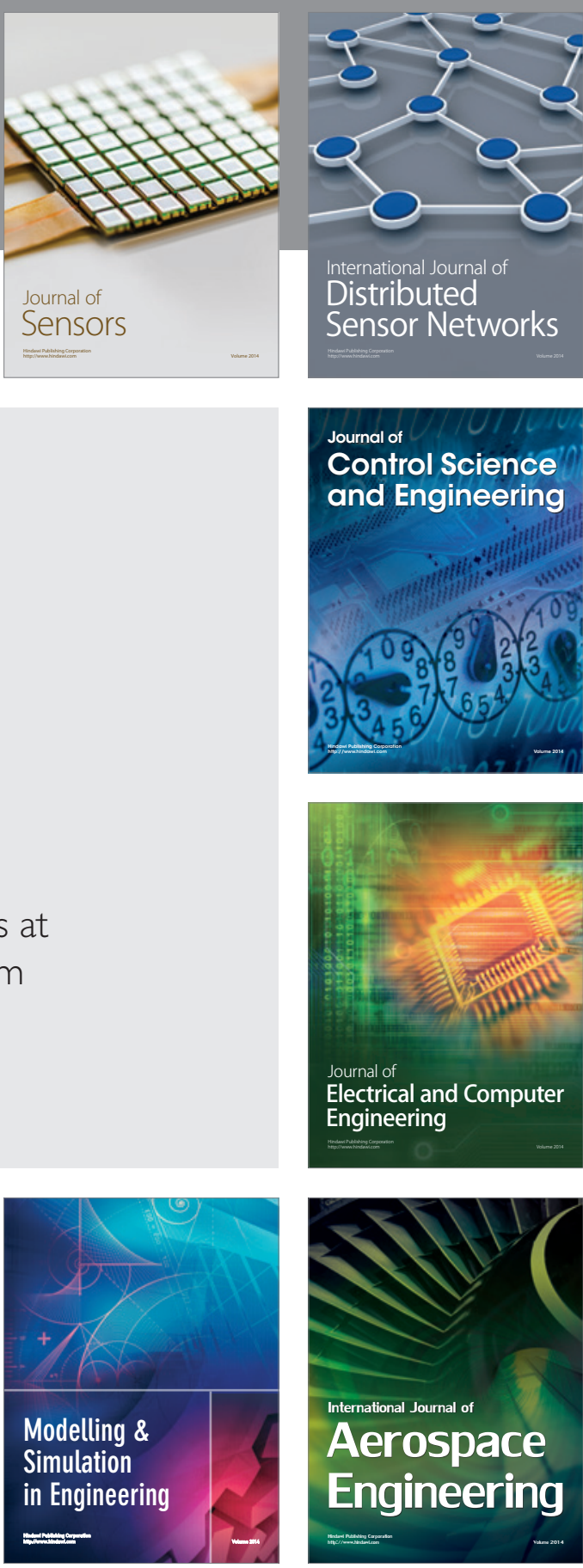

International Journal of

Distributed

Sensor Networks

Journal of

Control Science

and Engineering
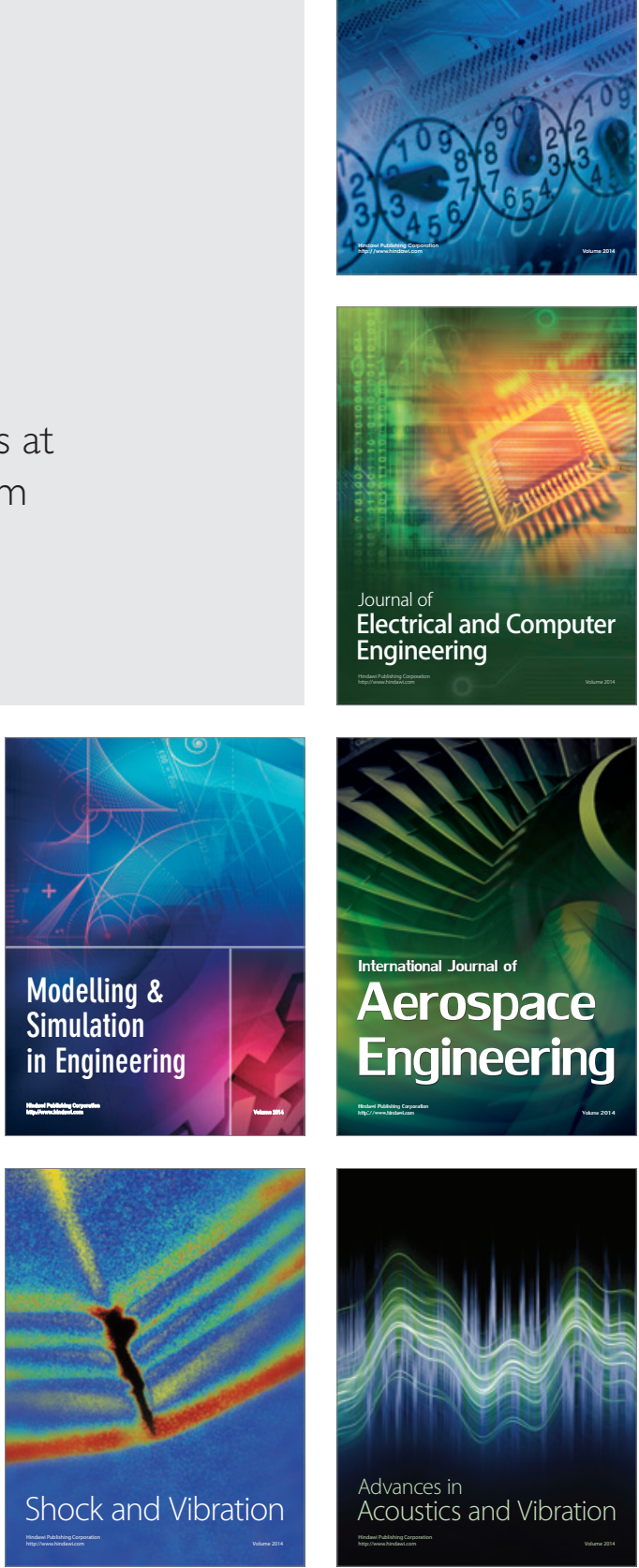